\section{Dementia

\title{
Microstructure of Strategic White Matter Tracts and Cognition in Memory Clinic Patients with Vascular Brain Injury
}

\author{
J. Matthijs Biesbroek ${ }^{a} \quad$ Alexander Leemans $^{b} \quad$ Hanna den Bakker $^{a}$ \\ Marco Duering ${ }^{c}$ Benno Gesierich ${ }^{c}$ Huiberdina L. Koek ${ }^{d}$ \\ Esther van den Berga, e Albert Postma ${ }^{\mathrm{e}}$ Geert Jan Biessels ${ }^{a}$ \\ on behalf of the Utrecht Vascular Cognitive Impairment ( $\mathrm{VCl}$ ) study group \\ ${ }^{a}$ Department of Neurology, Brain Center Rudolf Magnus, University Medical Center \\ Utrecht, Utrecht, The Netherlands; ${ }^{b}$ Image Sciences Institute, University Medical Center

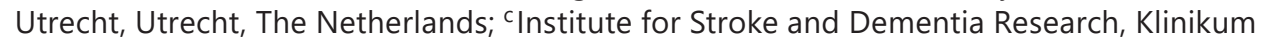 \\ der Universität München, Ludwig-Maximilians-Universität München, Munich, Germany; \\ ${ }^{\mathrm{d}}$ Department of Geriatrics, University Medical Center Utrecht, Utrecht, The Netherlands; \\ experimental Psychology, Helmholtz Institute, Utrecht University, Utrecht, The Netherlands
}

\section{Keywords}

Alzheimer disease - Diffusion tensor imaging $\cdot$ Small vessel disease $\cdot$ Strategic white matter tract $\cdot$ Vascular dementia

\begin{abstract}
Background: White matter injury is an important factor for cognitive impairment in memory clinic patients. We determined the added value of diffusion tensor imaging (DTI) of strategic white matter tracts in explaining variance in cognition in memory clinic patients with vascular brain injury. Methods: We included 159 patients. Conventional MRI markers (white matter hyperintensity volume, lacunes, nonlacunar infarcts, brain atrophy, and microbleeds), and fractional anisotropy and mean diffusivity (MD) of the whole brain white matter and of 18 white matter tracts were related to cognition using linear regression and Bayesian network analysis. Results: On top of all conventional MRI markers combined, MD of the whole brain white matter explained an additional 3.4\% $(p=0.014), 7.8 \%(p<0.001)$, and $1.2 \%(p=0.119)$ variance in executive functioning, speed, and memory, respectively. The Bayesian analyses of regional DTI measures identified strategic tracts for executive functioning (right superior longitudinal fasciculus), speed (left corticospinal tract), and memory (left uncinate fasciculus). MD within these tracts explained an additional 3.4\% $(p=0.012), 3.8 \%(p=0.007)$, and $2.1 \%(p=$ 0.041 ) variance in executive functioning, speed, and memory, respectively, on top of all con-
\end{abstract}


Biesbroek et al.: Microstructure of Strategic White Matter Tracts and Cognition in Memory Clinic Patients with Vascular Brain Injury

ventional MRI and global DTI markers combined. Conclusion: In memory clinic patients with vascular brain injury, DTI of strategic white matter tracts has a significant added value in explaining variance in cognitive functioning.

(C) 2018 The Author(s)

Published by S. Karger AG, Basel

\section{Introduction}

Brain MRI is often part of the diagnostic workup in memory clinic patients [1]. Yet, it is often difficult to relate the cognitive deficits of patients to MRI-visible markers of brain injury, in particular atrophy and vascular lesions [2]. This might be due to the fact that these visible lesions represent end-stage brain injury and do not capture early structural changes in the brain [2]. White matter microstructure, which can be determined with diffusion tensor imaging (DTI), is an emerging and more sensitive marker for brain injury, both in patients with a primary neurodegenerative disorder such as Alzheimer disease (AD) and - possibly even more so - in patients with vascular brain injury.

Previous studies have shown that the whole brain white matter microstructure is associated with cognitive functioning, independent of conventional MRI markers for brain injury (atrophy, white matter hyperintensities [WMHs], lacunes, and infarcts) in healthy communitydwelling individuals, and in patients with cognitive impairment due to either vascular injury or AD [3-8]. It is likely that the functional impact of disturbances in the white matter microstructure depends on their location, as is the case for visible vascular lesions $[9,10]$, which would imply that the microstructural characteristics of "strategic" white matter tracts explain more variance in cognitive functioning than does the global white matter microstructure. Thus, assessing the strategic white matter tract microstructure might improve the extent to which MRI markers can help to explain the cognitive profiles of memory clinic patients with vascular brain injury. However, this hypothesis has not yet been tested.

In this study, we therefore determined the added value of DTI-based assessment of strategic white matter tracts in explaining variance in cognitive functioning in memory clinic patients with vascular injury. We determined if (1) cognitive functioning is related to the microstructural characteristics of domain-specific strategic white matter tracts, and (2) the microstructural characteristics of these strategic white matter tracts have added value in explaining variance in cognitive functioning on top of conventional MRI markers for brain injury and the global white matter microstructure.

\section{Subjects and Methods}

Ethics

The study was approved by the Institutional Review Board of the University Medical Center Utrecht (UMCU). All patients provided informed consent prior to research-related procedures.

\section{Participants}

The participants were selected from patients included in the vascular cognitive impairment (VCI) cohort of the UMCU from 2009 through October 16, 2013 [11]. This cohort consists of consecutive patients who underwent a standardized evaluation at the memory clinic of either the Department of Geriatrics or the Department of Neurology of the UMCU and had vascular brain injury on MRI (i.e., possible VCI) [11]. No minimal thresholds for cognitive impairment or specific patterns of vascular brain injury were defined, in order to capture the whole spectrum of patients presenting to a memory clinic with cognitive complaints and visible vascular injury on MRI. 
Biesbroek et al.: Microstructure of Strategic White Matter Tracts and Cognition in

Memory Clinic Patients with Vascular Brain Injury

Vascular injury on MRI was operationalized as: either (1) substantial vascular disease on MRI (defined as either WMHs of Fazekas grade $\geq 2$ [12], a [lacunar] infarct, and/or $\geq 1$ cerebral microbleeds), or (2) mild WMHs (Fazekas grade 1) combined with 2 or more vascular risk factors (see online suppl. Methods for details; for all online suppl. material, see www.karger.com/doi/10.1159/000485376), or (3) a prior diagnosis of ischemic stroke or intracerebral hemorrhage. Also, patients were not selected based on evidence for the absence or presence of possible coexisting neurodegenerative etiologies, in line with the AHA/ASA criteria for VCI [1]. This nonrestrictive approach allowed us to study the full spectrum of cognitive disorders in relation to vascular brain injury as seen in a memory clinic setting. Patients with a primary nonvascular and nonneurodegenerative etiology (e.g., brain tumor, hydrocephalus, and excessive alcohol consumption) were not included in the cohort. We excluded 19 patients with insufficient DTI/MRI assessment, 1 patient with insufficient neuropsychological data, and 13 patients in whom the reconstruction of $\geq 1$ white matter tract(s) failed (which was caused by complete interruption of a tract by a lacune in 2 patients), resulting in the final inclusion of 159 patients.

\section{Neuropsychological Testing}

All patients underwent a standardized diagnostic workup at the memory clinic (see online suppl. Methods for details). Domain scores were formed for executive functioning, psychomotor speed, and verbal memory. Z-scores for executive functioning were computed by averaging the $z$-scores (calculated using the mean and SD within the study cohort) of (1) semantic verbal fluency (animals, 60 s) [13], (2) the Trail Making Test (TMT) B/A index (calculated with the equation [B - A]/A) [14], and (3) Stroop interference scores (calculated with the equation [Stroop III - (mean I and II)]/[mean I and II]) [15]. Z-scores for psychomotor speed were computed by averaging the $z$-scores of (1) the Letter Digit Coding Test (LDCT) [16], or (2) the Digit Symbol Test (DST) of the WAIS-III [17], (3) TMT part A, and (4) Stroop Tasks I and II. Z-scores for verbal memory were computed by averaging the $z$-scores of three measures of the Dutch version of the Rey Auditory Verbal Learning test [18]: (1) the sum of 5 immediate recall trials, (2) the delayed recall trial, and (3) the recognition trial.

$Z$-scores of tests in which high scores indicate poor performance were inverted. Missing variables were not included in the formation of the domain scores. The level of education was scaled according to the coding system of Verhage, where a score of 1 corresponds to not having finished primary school, and a score of 7 represents a university degree [19].

\section{MRI Scan Acquisition}

MRI data were acquired on a Philips 3.0-T scanner (Intera; Philips, Best, The Netherlands) using a standardized protocol as described previously [20]. In summary, diffusion MRI data were obtained using a singleshot spin echo-planar imaging sequence (TR/TE: 6,638/73 ms; 48 contiguous slices; reconstructed voxel size: $1.72 \times 1.72 \times 2.50 \mathrm{~mm}$ ), a flip angle of $90^{\circ}, 45$ isotropically distributed diffusion-sensitizing gradients with a b-value of $1,200 \mathrm{~s} / \mathrm{mm}^{2}$, and one $\mathrm{b}=0 \mathrm{~s} / \mathrm{mm}^{2}$. Other sequences included T2*-weighted (TR/TE: 1,653/20 ms; reconstructed voxel size: $0.96 \times 0.95 \times 3$ mm), FLAIR (TR/TE/TI: 11,000/125/2,800 ms; reconstructed voxel size: $0.96 \times 0.95 \times 3 \mathrm{~mm}$ ), and 3D T1-weighted sequences (TR/TE: 7.2/2.9 ms; reconstructed voxel size: $1.0 \times 1.0 \times 1.0 \mathrm{~mm}$ ).

\section{Image Processing}

All image processing steps were performed by experienced raters who were blinded to the clinical data. Microbleeds, lacunes, and nonlacunar infarcts were rated according to the STRIVE criteria [21]. WMHs, lacunes, and nonlacunar infarcts were manually segmented using in-house-developed software based on MeVisLab (MeVis Medical Solutions AG, Bremen, Germany) [22]. The interobserver reliability of WMH segmentation was good to excellent (Dice similarity coefficient of 0.75 [SD 0.14] and intraclass correlation coefficient [absolute agreement] of 0.98 , based on segmentation of 22 randomly selected scans by 2 experienced raters).

Total brain volume and intracranial volume were quantified using the Statistical Parametric Mapping $12 \mathrm{~b}$ unified segmentation approach and used to compute the brain parenchymal fraction (brain volume/ intracranial volume) as a measure of brain atrophy [23]. Diffusion MRI data were corrected for subject motion and eddy current distortions with ExploreDTI (www.exploredti.com) [24, 25]. The following data processing steps were subsequently performed: (1) diffusion MR images were registered to the corresponding T1 image to correct for echo-planar imaging deformations [26]; (2) FLAIR images and corresponding lesion maps were registered to the corresponding T1 image; (3) whole brain fiber tracking was 
performed based on constrained spherical deconvolution, with the parameter setting and regions of interest as defined previously $[27,28]$; (4) the results of the whole brain fiber tracking were used to extract the fractional anisotropy (FA)/mean diffusivity (MD) of the whole brain white matter, normal-appearing white matter, and total WMH volume (expressed as the proportion of whole brain white matter affected by WMH); (5) 18 white matter tracts were reconstructed for each patient using previously described methods [29]: the forceps minor and major, anterior thalamic radiation, inferior and superior longitudinal fasciculus, cingulum of the cingulate gyrus and hippocampus, inferior fronto-occipital fasciculus, and uncinate fasciculus for each hemisphere (see online suppl. Methods for details); and (6) the mean FA/MD and the proportion that was affected by WMHs or infarcts/lacunes were extracted for each white matter tract (lacunar and nonlacunar infarct volumes were combined into a single variable). These six steps are illustrated in Figure 1.

Statistics

We tested the following two complementary hypotheses.

Hypothesis 1

The impact of disturbances in white matter microstructure on cognitive functioning depends on their location (i.e., cognitive functioning is related to the microstructural characteristics of domain-specific strategic white matter tracts). First, Bayesian network analyses were used to analyze the conditional dependencies between FA/MD in the 18 white matter tracts, age, sex, and education as potential determinants, and executive functioning, psychomotor speed, and verbal memory as outcome variables (bnlearn R package [30]; settings described in Duering et al. [31]).

In brief, Bayesian network analyses identify the variables with a deterministic influence on the (cognitive) outcome variables. The main advantage of Bayesian network analysis is that it deals well with multicollinearity. It separates variables with a direct deterministic influence on the outcome variables from other variables that, although showing a correlation with the outcome variable, have only an indirect influence when taking the direct determinants into account. The latter variables are therefore considered conditionally independent from the outcome variables. Bayesian network analysis produces networks in which direct determinants are connected directly to the outcome variables, while conditionally independent variables are connected only indirectly, via other variables.

The strength of the connections between direct determinants and cognitive variables was assessed by 100 bootstrap replications. We additionally performed a supplementary linear regression analysis to verify the results of the Bayesian network analyses in which the FA/MD of each tract was related to each cognitive domain, followed by a Bonferroni correction for 36 comparisons.

Hypothesis 2

The microstructural characteristics of strategic white matter tracts have added value in explaining variance in cognitive functioning on top of conventional MRI markers and global white matter microstructural characteristics. First, multivariate multiple linear regression analyses were performed with the $z$-score for each cognitive domain as the dependent variable. Age, sex, level of education, and conventional MRI markers (WMH volume, brain parenchymal fraction, and presence of lacunes, nonlacunar infarcts, and microbleeds) were first entered as independent variables, to verify the relation between global white matter microstructural characteristics and cognition, as known from the literature [3, 5-8]. FA/MD of the whole brain white matter and normal-appearing white matter were then added as independent variables to assess their added value in explaining variance in cognitive functioning. Next, DTI measures of the white matter tracts that were identified as strategic tracts in the Bayesian analysis (defined as a connection with a cognitive domain with a resampling frequency $>50 \%$ in the bootstrapping replications) were added to the regression model, to assess their added value. Additionally, a stepwise backward linear regression analysis was performed to see which of the variables in the final model would be removed, indicating that their contribution to cognitive performance is fully captured by the variables that do remain in the model. This was done because it is likely that some MRI markers (such as global WMHs) become redundant when more sensitive markers (such as microstructural characteristics of the white matter) are added to the model. Finally, sensitivity analyses were performed in which the backward linear regression analysis was repeated in the following subgroups of patients: (1) only patients with vascular dementia, $\mathrm{MCI}$, or no cognitive impairment (i.e., after the exclusion of patients with a diagnosis of dementia due to $\mathrm{AD}$ or other neurodegenerative etiologies); (2) only patients with vascular dementia or MCI (i.e., after additional exclusion of patients with no cognitive impairment); and (3) only patients with dementia due to AD. 
Dementia

and Geriatric

Cognitive Disorders
Dement Geriatr Cogn Disord 2017;44:268-282

DOI: $10.1159 / 000485376$

(c) 2018 The Author(s). Published by S. Karger AG, Basel www.karger.com/dem

Biesbroek et al.: Microstructure of Strategic White Matter Tracts and Cognition in Memory Clinic Patients with Vascular Brain Injury
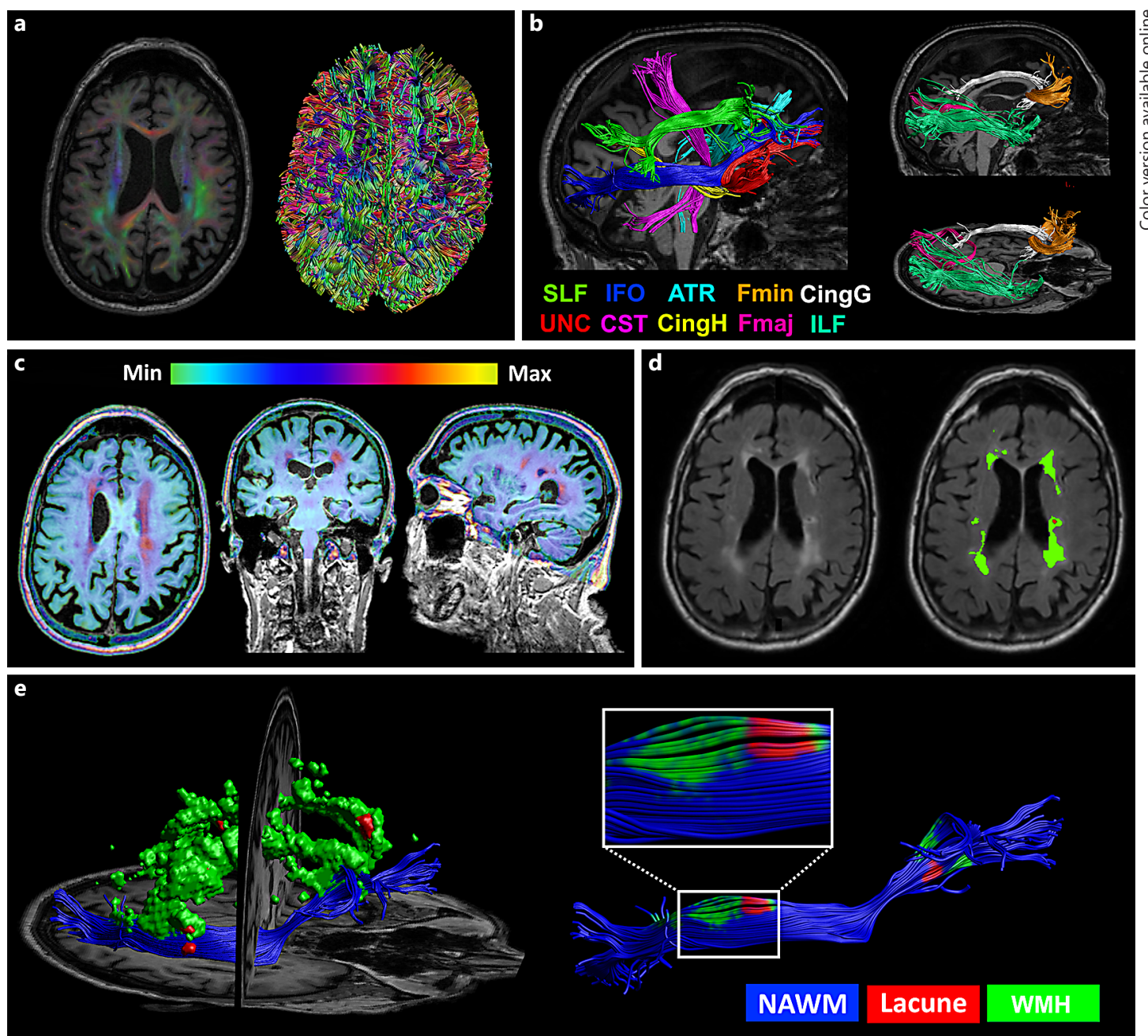

Fig. 1. Illustration of the image processing procedure and integration of microstructural and macrostructural data for a single patient. a Left: color-coded fractional anisotropy (FA) map overlaying the corresponding T1 image after correcting for susceptibility-induced deformations. Right: results of whole brain tractography, which is used to extract the FA and mean diffusivity (MD) of the whole brain white matter. $\mathbf{b}$ Tractography results of 10 white matter fiber bundles ( 8 of which were bilaterally reconstructed), used to extract the FA and MD of each tract. c T2 FLAIR image projected onto the corresponding T1 image after rigid registration. The color bar applies to the intensity range of the T2 FLAIR image. d Sagittal T2 FLAIR slice before and after manual segmentation. White matter hyperintensities (WMHs) are green and lacunes are red. e Left: 3D reconstruction of WMHs and lacunes in relation to the right inferior fronto-occipital fasciculus. Right: integration of WMHs and lacunes with the inferior fronto-occipital fasciculus is used to extract the FA and MD of the normal-appearing white matter and the proportion of the tract that is affected by these lesions.

\section{Results}

The clinical and imaging characteristics of the 159 included patients are shown in Table 1. The cognitive profile of the study cohort is provided in online supplementary Table 1. Out of 2,862 reconstructed white matter tracts, 153 were affected by lacunes or nonlacunar infarcts; these affected tracts were of similar volume to their contralateral, unaffected counterparts (mean volume of 6.0 and $5.9 \mathrm{~mL}$, respectively; $p=0.75$ ), but MD was higher (mean MD of $0.95 \times 10^{-3}$ and $0.88 \times 10^{-3} \mathrm{~mm}^{2} / \mathrm{s}$, respectively; $p<0.001$ ). 
Table 1. Characteristics of the study cohort $(n=159)$

$\begin{array}{lc}\text { Mean age } \pm \text { SD, years } & 72.4 \pm 10.1 \\ \text { Male, } \% & 55 \\ \text { Median years of education (range) } & 5(1-7) \\ \text { Vascular risk factors, \% } & \\ \quad \text { Hypertension } & 96 \\ \text { Hyperlipidemia } & 72 \\ \text { Current smoking } & 15 \\ \text { Diabetes mellitus } & 38 \\ \text { Obesity } & 19 \\ \text { History of manifest arterial disease } & 21 \\ \text { Diagnosis, \% } & \\ \text { No objective cognitive impairment } & 17 \\ \text { Mild cognitive impairment } & 35 \\ \text { Vascular dementia } & 6 \\ \text { Alzheimer disease } & 36 \\ \text { Dementia - other }{ }^{\mathrm{a}} & 6 \\ \text { Imaging characteristics } & \\ \text { Patients with lacunes, } \% & 28 \\ \text { Patients with nonlacunar infarcts, } \% & 20 \\ \text { Patients with microbleeds, } \%{ }^{\mathrm{b}} & 42 \\ \text { Median total WMH volume (range) as } \% \text { of WBWM } & 1.77(0.02-28.51) \\ \text { Mean brain parenchymal fraction } \pm \mathrm{SD}^{\mathrm{c}} & 0.66 \pm 0.07 \\ \text { Mean FA WBWM } \pm \text { SD (range) } & 0.380 \pm 0.026(0.279-0.433) \\ \text { Mean MD WBWM } \pm \text { SD (range), } \times 10^{-3} \mathrm{~mm}^{2} / \mathrm{s} & 0.979 \pm 0.062(0.829-1.151)\end{array}$

WMH, white matter hyperintensity; WBWM, whole brain white matter; FA, fractional anisotropy; MD, mean diffusivity. a Primary progressive aphasia, cortical basal syndrome, progressive supranuclear palsy, frontotemporal dementia, and 1 case of a delayed diagnosis pending further examinations. ${ }^{\text {b }}$ Data missing in 3 cases. ${ }^{\mathrm{c}}$ Measure for brain atrophy, calculated by dividing total brain volume by intracranial volume.

\section{Identification of Strategic White Matter Tracts}

The Bayesian network analyses identified MD and FA in the right superior longitudinal fasciculus as a direct determinant of executive functioning, MD in the left corticospinal tract as a determinant of psychomotor speed, and MD of the left uncinate fasciculus as a determinant of verbal memory (Fig. 2). The supplementary linear regression analysis in which the FA/MD of all 18 white matter tracts were related to each cognitive domain confirmed that MDs of the white matter tracts identified in the Bayesian models were indeed the strongest determinants of their corresponding cognitive domain (online suppl. Table 2), and that these correlations were independent of WMH and infarct volumes within these tracts (Table 2).

\section{Added Value of the Strategic White Matter Tract Microstructure over Conventional MRI Markers}

As a first step, the variance in cognition explained by demographics, conventional MRI markers, and whole brain DTI measures was assessed. Age, sex, and education explained $6.5 \%$ of the variance in executive functioning, $8.7 \%$ of the variance in psychomotor speed, and $21.0 \%$ of the variance in verbal memory (Table 3, model 1). All conventional MRI markers combined (WMH volume, brain parenchymal fraction, lacunes, nonlacunar infarcts, and microbleeds) explained an additional $11.4 \%(p=0.002)$ of the variance in executive functioning, $8.0 \%(p=0.021)$ of the variance in psychomotor speed, and $5.6 \%(p=0.056)$ of the variance in verbal memory (model 3). On top of that, MD of the whole brain white matter explained an additional 3.4\% ( $p=0.009)$ of the variance in executive functioning and $7.8 \%$ 
Dementia

and Geriatric

Cognitive Disorders

Dement Geriatr Cogn Disord 2017:44:268-282

$(p<0.001$ ) of the variance in psychomotor speed (model $4 \mathrm{~b}$ and $4 \mathrm{~d}$ ), whereas FA/MD of the whole brain white matter did not improve the model for verbal memory.

In the next step, we determined whether the microstructural characteristics of the identified strategic white matter tracts were of added value. Adding the microstructural characteristics of the strategic white matter tracts to the model including age, sex, education, all

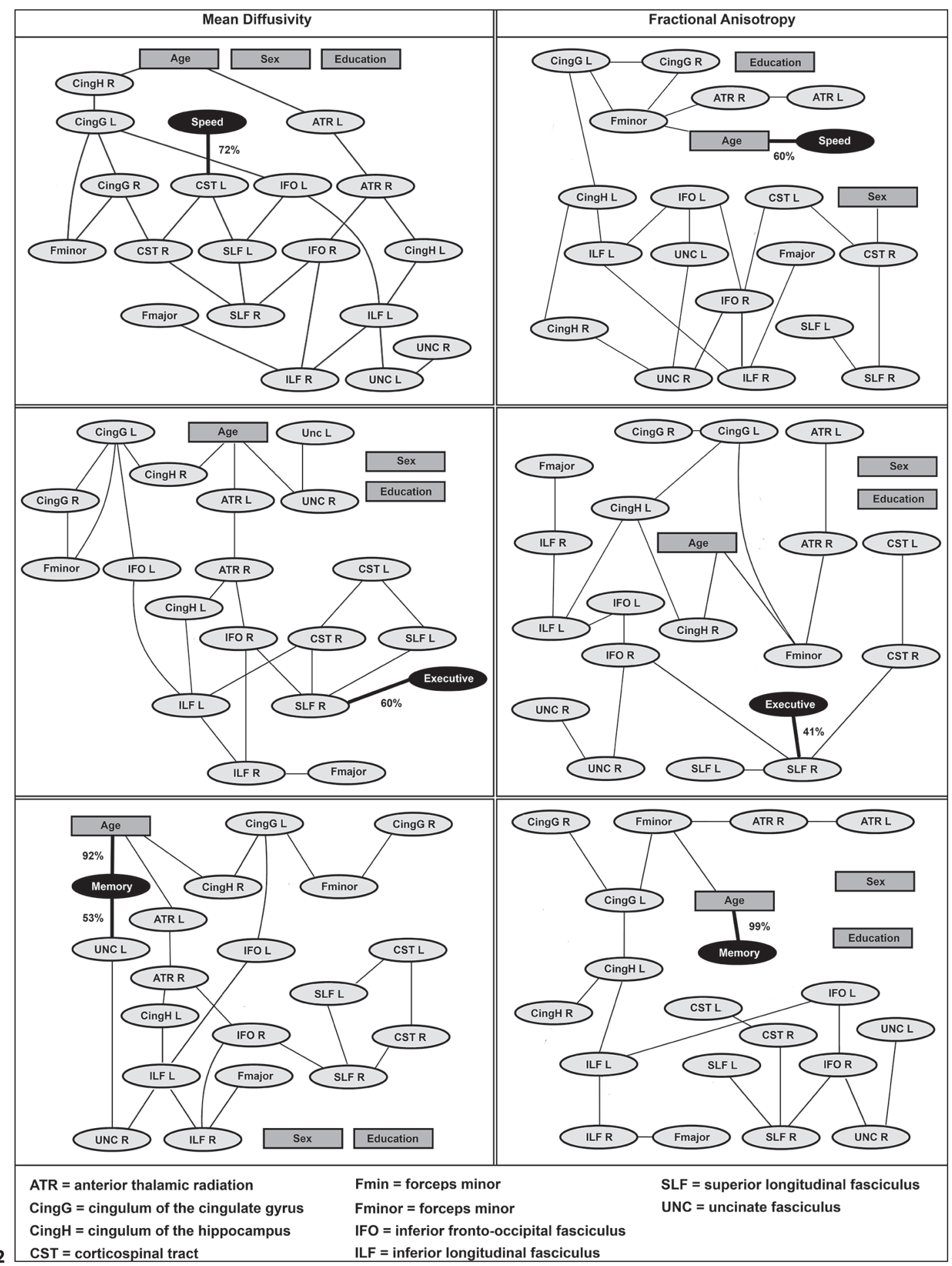

(For legend see next page.) 
Biesbroek et al.: Microstructure of Strategic White Matter Tracts and Cognition in Memory Clinic Patients with Vascular Brain Injury

combined conventional MRI markers, and global DTI markers (FA/MD of the whole brain white matter) (Table 3, model 5) further increased the explained variance in cognitive functioning. MD in the right superior longitudinal fasciculus explained an additional $2.2 \%(p=$ 0.044 ) of the variance in executive functioning, MD in the left corticospinal tract explained an additional $3.8 \%$ of the variance in psychomotor speed ( $p=0.006)$, and MD in the left uncinate fasciculus explained an additional $2.1 \%(p=0.041)$ of the variance in verbal memory (Table 3 , model 6).

Finally, we performed stepwise backward linear regression analyses to establish for each cognitive domain which variables remained relevant and which became redundant when all variables were considered together (i.e., age, sex, education, all conventional MRI markers, MD and FA of the whole brain white matter, and MD of the strategic white matter tracts) (Table 4). For executive functioning, MD of the right superior longitudinal fasciculus, presence of lacunes, and brain parenchymal fraction remained in the model. For psychomotor speed, MD of the left corticospinal tract, MD of the whole brain white matter, and education remained

Table 2. Identification of strategic white matter tracts: correlation between microstructure and cognition, independent of regional WMH and infarct volumes

\begin{tabular}{|c|c|c|c|c|}
\hline Model & Independent variables & $R^{2}$ & $p$ value $R^{2}$ & $\beta(95 \% \mathrm{CI})$ \\
\hline \multicolumn{5}{|c|}{ Executive functioning ( $n=156)$} \\
\hline 1 & Age, sex, and education & 0.065 & $0.016^{*}$ & - \\
\hline 2 & Model $1+$ WMH and infarct volume right SLF & 0.106 & $0.035^{*}$ & - \\
\hline $3 a$ & Model $2+$ MD right SLF & 0.190 & $<0.001^{*}$ & $-0.46(-0.70$ to -0.23$)$ \\
\hline $3 \mathrm{~b}$ & Model $2+$ MD right SLF NAWM & 0.199 & $<0.001^{*}$ & $-0.37(-0.55$ to -0.19$)$ \\
\hline \multicolumn{5}{|c|}{ Psychomotor speed $(n=156)$} \\
\hline 1 & Age, sex, and education & 0.087 & $0.003^{*}$ & - \\
\hline 2 & Model $1+$ WMH and infarct volume left CST & 0.167 & $<0.001^{*}$ & - \\
\hline $3 a$ & Model $2+$ MD left CST & 0.241 & $<0.001^{*}$ & $-0.32(-0.49$ to -0.15$)$ \\
\hline $3 b$ & Model $2+$ MD left CST NAWM & 0.242 & $<0.001^{*}$ & $-0.29(-0.44$ to -0.14$)$ \\
\hline \multicolumn{5}{|c|}{ Verbal memory $(n=156)$} \\
\hline 1 & Age, sex, and education & 0.210 & $<0.001^{*}$ & - \\
\hline 2 & Model $1+$ WMH and infarct volume left UNC & 0.216 & 0.544 & - \\
\hline $3 a$ & Model 2 + MD left UNC & 0.271 & $0.001^{*}$ & $-0.28(-0.44$ to -0.11$)$ \\
\hline $3 b$ & Model 2 + MD left UNC NAWM & 0.271 & $0.001^{*}$ & $-0.26(-0.41$ to -0.11$)$ \\
\hline
\end{tabular}

The white matter tracts in these analyses are selected based on the results of the Bayesian network analysis. WMH, white matter hyperintensity; MD, mean diffusivity; SLF, superior longitudinal fasciculus; NAWM, normal-appearing white matter; CST, corticospinal tract; UNC, uncinate fasciculus.

Fig. 2. Bayesian networks for executive functioning (executive), psychomotor speed (speed), and verbal memory (memory). Bayesian network analyses were used to analyze the conditional dependencies between FA/MD in 18 white matter tracts, age, sex, and education as potential determinants, and executive functioning, psychomotor speed, and verbal memory as outcome variables. Variables that are directly connected to one of the cognitive domains are identified as direct determinants. Variables that are connected indirectly to the cognitive domains (via other variables) are conditionally independent. As such, this method separates determinants with a direct deterministic influence on the outcome variable from other determinants that, although showing a univariate correlation with the outcome variable, have only an indirect influence when taking the direct determinants into account. Percentages indicate the confidence level of the arcs towards cognitive domains determined by 100 bootstrap replications. L, left; $\mathrm{R}$, right. 
Table 3. Additional value of global and regional DTI markers in explaining variance in cognitive functioning on top of conventional MRI markers

\begin{tabular}{|c|c|c|c|c|c|c|c|c|c|c|}
\hline \multirow[t]{2}{*}{$\begin{array}{l}\text { Mod- } \\
\text { el }\end{array}$} & \multirow[t]{2}{*}{ Independent variables } & \multicolumn{3}{|c|}{$\begin{array}{l}\text { Executive functioning } \\
(n=156)\end{array}$} & \multicolumn{3}{|c|}{$\begin{array}{l}\text { Psychomotor speed } \\
(n=156)\end{array}$} & \multicolumn{3}{|c|}{$\begin{array}{l}\text { Verbal memory } \\
(n=156)\end{array}$} \\
\hline & & $R^{2}$ & $p$ value & $\beta$ & $R^{2}$ & $p$ value & $\beta$ & $R^{2}$ & $p$ value & $\beta$ \\
\hline 1 & Age, sex, and education & 0.065 & $0.016^{*}$ & - & 0.087 & $0.003^{*}$ & - & 0.210 & $<0.001$ & - \\
\hline $2 \mathrm{a}$ & Model 1 + total WMH volume & 0.101 & $0.016^{*}$ & -0.19 & 0.108 & 0.062 & -0.15 & 0.218 & 0.223 & -0.089 \\
\hline $2 b$ & Model $1+$ presence of lacunes & 0.068 & 0.553 & -0.05 & 0.104 & 0.097 & -0.14 & 0.211 & 0.573 & 0.043 \\
\hline $2 c$ & Model $1+$ presence of microbleeds ${ }^{\mathrm{a}}$ & 0.067 & 0.651 & -0.04 & 0.094 & 0.293 & -0.08 & 0.214 & 0.388 & 0.064 \\
\hline $2 \mathrm{~d}$ & Model $1+$ presence of nonlacunar infarcts & 0.087 & 0.063 & -0.15 & 0.102 & 0.115 & -0.12 & 0.212 & 0.520 & -0.047 \\
\hline $2 \mathrm{e}$ & Model $1+$ brain atrophy (BPF) & 0.134 & $0.001^{*}$ & 0.31 & 0.145 & $0.002^{*}$ & 0.29 & 0.239 & $0.017^{*}$ & 0.205 \\
\hline $2 \mathrm{f}$ & Model 1 + FA WBWM & 0.129 & $0.001^{*}$ & 0.26 & 0.155 & $0.001^{*}$ & 0.26 & 0.215 & 0.304 & 0.075 \\
\hline $2 g$ & Model $1+$ MD WBWM & 0.180 & $<0.001^{*}$ & -0.37 & 0.232 & $<0.001^{*}$ & -0.41 & 0.245 & $0.009 *$ & -0.204 \\
\hline 3 & Model $1+$ all conventional MRI markers ${ }^{\mathrm{b}}$ & 0.179 & $0.002^{*}$ & - & 0.167 & $0.021 *$ & - & 0.266 & 0.056 & - \\
\hline $4 a$ & Model 3 + FA WBWM & 0.200 & 0.054 & 0.19 & 0.199 & $0.018^{*}$ & 0.23 & 0.267 & 0.709 & 0.034 \\
\hline $4 \mathrm{~b}$ & Model 3 + MD WBWM & 0.213 & $0.014^{*}$ & -0.29 & 0.245 & $<0.001^{*}$ & -0.44 & 0.278 & 0.119 & -0.175 \\
\hline $4 c$ & Model 3 + FA whole brain NAWM & 0.200 & 0.051 & 0.18 & 0.199 & $0.018^{*}$ & 0.23 & 0.267 & 0.758 & 0.027 \\
\hline $4 d$ & Model 3 + MD whole brain NAWM & 0.217 & $0.009^{*}$ & -0.29 & 0.245 & $<0.001^{*}$ & -0.41 & 0.279 & 0.107 & -0.168 \\
\hline 5 & Model 3 + FA and MD WBWM & 0.213 & $0.050^{*}$ & - & 0.248 & $0.001^{*}$ & - & 0.288 & 0.120 & - \\
\hline $6 a$ & Model $5+$ MD right SLF & 0.236 & $0.040^{*}$ & -0.32 & - & - & - & - & - & - \\
\hline $6 b$ & Model 5 + MD left CST & - & - & - & 0.286 & $0.007^{*}$ & -0.25 & - & - & - \\
\hline $6 c$ & Model $5+$ MD left uncinate fasciculus & - & - & - & - & - & - & 0.309 & $0.041^{*}$ & -0.165 \\
\hline
\end{tabular}

In these analyses, each conventional MRI marker (model 2a-e) and global DTI marker (model 2f, g) was first considered separately. Next, the added value of global DTI markers was determined (model $4 \mathrm{a}-\mathrm{d}$ ). Finally, the added value of the strategic tract (as identified in the Bayesian analyses) microstructure on top of all global conventional MRI markers and DTI markers was determined (model 6a-c). WMH, white matter hyperintensity; BPF, brain parenchymal fraction; FA, fractional anisotropy; MD, mean diffusivity; WBWM, whole brain white matter; NAWM, normal-appearing white matter; SLF, superior longitudinal fasciculus; CST, corticospinal tract. ${ }^{\mathrm{a}}$ Data on microbleeds missing in 3 cases ${ }^{\mathrm{b}}$ Total WMH volume, the presence of lacunes, nonlacunar infarcts, and microbleeds, and BPF.

Table 4. Stepwise backward linear regression analyses

\begin{tabular}{lcc}
\hline Independent variables & $p$ value & $\beta(95 \%$ CI $)$ \\
\hline Executive functioning $\left(R^{2}=0.215\right)$ & & \\
$\quad$ MD right SLF & $<0.001$ & $-0.39(-0.56$ to -0.22$)$ \\
$\quad$ Brain atrophy (BPF) & 0.027 & $0.18(0.02$ to 0.34$)$ \\
$\quad$ Presence of lacunes & 0.020 & $0.19(0.03$ to 0.34$)$ \\
Psychomotor speed $\left(R^{2}=0.246\right)$ & & \\
MD left CST & 0.053 & $-0.18(-0.36$ to 0.00$)$ \\
MD WBWM & $<0.001$ & $-0.35(-0.53$ to -0.17$)$ \\
Education & 0.083 & $0.12(-0.02$ to 0.27$)$ \\
Verbal memory $\left(R^{2}=0.276\right)$ & 0.019 & $-0.19(-0.34$ to -0.03$)$ \\
$\quad$ MD left uncinate fasciculus & 0.003 & $-0.25(-0.42$ to -0.09$)$ \\
Age & 0.068 & $0.13(-0.01$ to 0.27$)$ \\
Education & 0.012 & $-0.34(-0.60$ to -0.08$)$ \\
MD WBWM & 0.038 & $-0.25(-0.48$ to -0.01$)$ \\
FA WBWM &
\end{tabular}

The final multivariable model and corresponding $R^{2}$ of the stepwise backward linear regression analyses for each cognitive domain are shown. Independent variables entered in the model were age, sex, education, all conventional MRI markers (WMH volume, lacunes, nonlacunar infarcts, microbleeds, and brain atrophy), FA and MD of the whole brain white matter, and MD of strategic white matter tracts (the right SLF for executive functioning, the left CST for psychomotor speed, and the left uncinate fasciculus for verbal memory). MD of these strategic white matters tracts remained in the model, whereas many conventional MRI markers were excluded. MD, mean diffusivity; SLF, superior longitudinal fasciculus; BPF, brain parenchymal fraction; CST, corticospinal tract; WBWM, whole brain white matter; FA, fractional anisotropy. 
Biesbroek et al.: Microstructure of Strategic White Matter Tracts and Cognition in Memory Clinic Patients with Vascular Brain Injury

in the model. For verbal memory, MD of the left uncinate fasciculus, FA and MD of the whole brain white matter, age, and education remained in the model.

In the sensitivity analyses, after the exclusion of patients with a dementia diagnosis due to AD or other neurodegenerative etiologies (online suppl. Table 3) the results were essentially the same. MD of the right superior longitudinal fasciculus remained in the model for executive functioning, MD of the left uncinate fasciculus remained in the model for verbal memory, and MD of the left corticospinal tract remained in the model for visuomotor speed (online suppl. Table 3). A sensitivity analysis after additional exclusion of patients with no cognitive impairment - thereby creating a cohort of patients $(n=67)$ with vascular brain injury, objective cognitive impairment, and no primary diagnosis of dementia due to neurodegenerative disease - also generated the same results (online suppl. Table 4). In the sensitivity analysis including only patients with a diagnosis of AD dementia, MD of the right superior longitudinal fasciculus remained in the model for executive functioning (but with a lower explained variance; see online suppl. Table 5), whereas both global and regional DTI measures did not remain in the models for visuomotor speed and memory.

\section{Discussion}

This study shows that in memory clinic patients with vascular injury (1) the impact of disturbances in white matter microstructure on cognitive functioning depends on their location, and (2) the microstructural characteristics of strategic (cognitive domain-specific) white matter tracts have added value in explaining variance in cognitive functioning on top of all conventional MRI markers of brain injury and the global white matter microstructural characteristics combined.

The current study is the first to perform a fully integrated analysis considering both conventional MRI markers (i.e., visible vascular lesions and brain atrophy) and the microstructural characteristics of both the whole brain white matter and strategic white matter tracts in a single statistical model. Previous studies focused on DTI without taking visible lesions into account, or studied DTI measures and visible lesions at either the level of the whole brain white matter or the level of specific white matter tracts without taking global DTI measures into account $[3-8,32,33]$. By considering all measures together, we are able to demonstrate the true additional value of the strategic tract microstructure on top of all the other measures. Furthermore, advanced and robust Bayesian network analyses allowed for the identification of the main determinants of each cognitive domain in a hypothesis-free fashion, taking into account intercorrelations and multiple comparisons. The reproduction of the involvement of the thus identified tracts in each cognitive domain in the linear regression analyses, a method that is statistically independent of the Bayesian analyses, provides converging evidence for a strategic role of these tracts in these specific cognitive domains. Moreover, by applying a spherical deconvolution-based tractography approach, we have addressed the main limitation of the conventionally applied DTI-based tractography, which is not being able to reliably reconstruct pathways in complex white matter configurations, for example, with crossing fibers [27, 34, 35].

In the current study, memory clinic patients with vascular injury were included using nonrestrictive criteria. Because vascular injury in memory clinic patients often co-occurs with AD pathologies $[1,36]$, patients with cognitive impairment caused by a mixture of cerebral vascular disease and AD were also included in our study. This is in line with proposed criteria for VCI, which also include patients with co-occurring AD. In our cohort, $36 \%$ of the patients were diagnosed with dementia due to AD. In order to rule out that our findings are solely driven by AD-related neurodegenerative pathology, we performed a sensitivity analysis 
Dementia

Cognitive Disorders
Dement Geriatr Cogn Disord 2017;44:268-282

\begin{tabular}{l|l}
\hline DOI: $10.1159 / 000485376$ & (c) 2018 The Author(s). Published by S. Karger AG, Basel
\end{tabular} www.karger.com/dem

Biesbroek et al.: Microstructure of Strategic White Matter Tracts and Cognition in Memory Clinic Patients with Vascular Brain Injury

excluding patients with a clinical diagnosis of dementia due to AD or other neurodegenerative etiologies, for which the results were essentially the same as for the main analysis (online suppl. Table 3). When both patients with dementia due to AD or other neurodegenerative etiologies and patients with no cognitive impairment were excluded from the analyses, the results of the stepwise backward regression analyses again remained the same: MD of the identified strategic tracts remained in the final model, whereas most conventional MRI markers became redundant (online suppl. Table 4). These findings suggest that in memory clinic patients with vascular brain injury, the microstructural characteristics of strategic white matter tracts are functionally relevant MRI markers that explain variance in cognitive functioning, also after excluding patients with co-occurring $\mathrm{AD}$ and patients without cognitive impairment. Furthermore, in the sensitivity analysis in which the analyses were restricted to only patients with a diagnosis of dementia due to $\mathrm{AD}$, the association between MD in the right superior longitudinal fasciculus and executive functioning remained, albeit with a lower explained variance, and the associations between DTI measures and visuomotor speed and memory were not reproduced (online suppl. Table 5). The associations observed between white matter tract microstructure and cognitive functioning might therefore reflect vascular injury. However, it should be noted that these associations may not only be driven by vascular or neurodegenerative pathology, but might also reflect normal or "healthy" structure-function relations [37]. Hence, even when studied in the context of patients presenting with cognitive complaints and vascular brain injury, these DTI measures should not be considered as specific markers of vascular damage.

Previous studies have shown that adding DTI of the whole brain white matter to conventional MRI parameters is of additional value in explaining variance in cognitive functioning in patients with lacunar stroke and CADASIL syndrome and in healthy individuals with agerelated small vessel disease $[5-8,38]$. The reported variance explained by global DTI measures varied greatly depending on the studied population and analytic approach; in a study of 35 lacunar stroke patients, whole brain FA explained up to $40 \%$ of the variance in executive functioning (univariate analysis, not corrected for other variables) [5], whereas whole brain FA/ MD explained only $1 \%$ of the variance in cognitive functioning on top of conventional MRI parameters in a cohort study of 499 cognitively preserved community-dwelling individuals [6]. Our findings indicate that global DTI measures have a significant added value in memory clinic patients with vascular injury (who often have mixed pathologies, i.e., a combination of age-related small vessel disease and neurodegenerative disease), a clinically highly relevant population that had not been studied before in this context.

In the Bayesian network analysis (Fig. 2), we identified several cognitive domain-specific strategic white matter tracts. The observed crucial role of the left corticospinal tract in deficits in psychomotor speed is in line with findings from a previous study of patients with CADASIL syndrome in which the lacunar volume in this tract was among the most important determinants of processing speed (probably reflecting the motor component of this cognitive domain) [39]. A crucial role of the right superior longitudinal fasciculus in executive functioning, and of the left uncinate fasciculus in verbal memory, has also been previously suggested [32, 33, 40]. The microstructural characteristics of several additional tracts had a significant correlation with cognitive functioning (online suppl. Table 2), but they were not identified as direct determinants by the Bayesian network analysis. Possibly, the current sample size provided insufficient statistical power to demonstrate these connections, even though this study is the largest to apply a DTI-based assessment of individual tracts in memory clinic patients with vascular injury to date. Consequently, our results should not be interpreted as a complete map of relevant white matter tracts for executive functioning, psychomotor speed, and memory. Instead, the findings of this study provide proof for the concept that in memory clinic patients with vascular brain injury, the microstructural characteristics of strategic 
white matter tracts are functionally relevant MRI markers in explaining variance in cognitive functioning beyond what is explained by conventional MRI markers and the global white matter microstructure. Of note, 2 patients had to be excluded because $\geq 1$ tract(s) appeared to be completely interrupted by a lacune (the left anterior thalamic radiation in both patients, the bilateral inferior fronto-occipital fasciculus in one patient, and the left superior longitudinal fasciculus in one patient), which might have resulted in a slight underestimation of the impact of the microstructural characteristics of these tracts on cognitive functioning. The vast majority of tracts that were affected by lacunes or nonlacunar infarcts could still be reconstructed [20].

It is worth noting that though combining DTI measures with conventional MRI markers significantly improved the explained variance in cognitive functioning, a large part of the variance $(>70 \%)$ remained unexplained. It is likely that integrating conventional MRI markers and DTI markers with additional markers, such as measures for structural brain connectivity [41], brain amyloid imaging [42], microinfarcts [43], and additional demographic data (such as lifestyle factors $[1,44]$ ) may further increase the explained variance in cognitive functioning. Also, using quantitative measures for hippocampal [45], gray matter, and white matter atrophy (instead of using a single quantitative measure for global atrophy as was done in the current study) might help to further increase the explained variance in cognitive functioning. Assessing regional gray matter atrophy might be particularly interesting in relation to the DTI-based assessment of strategic white matter tracts, since subcortical vascular lesions are known to cause focal cortical thinning via secondary degeneration [46]. An effort to acquire and integrate all these data into a large cohort in order to push the explained variance in cognitive functioning to a maximum would be interesting, but also very challenging. Also, it should be noted that a certain amount of variance in cognitive functioning will probably always remain unexplained by demographics and MRI markers, because the spectrum of normal (innate and acquired) variability in brain structure and function cannot be fully captured by such markers, and because of within-person variability in cognitive test performance (i.e., performance on cognitive tests are known to vary to some extent from one moment to the next) [47].

Because we wanted to determine the added value of DTI of strategic white matter tracts on top of conventional MRI markers (WMH volume, infarcts, lacunes, microbleeds, and brain atrophy) and global DTI markers, a relatively high number of variables were included in this study. The rationale behind this approach is that these conventional MRI markers are known to be associated with cognitive functioning in memory clinic patients, frequently used, and more readily available than DTI-based tractography. We therefore think that a new MRI marker that requires considerable data processing, such as DTI-based tractography, should not only be univariately associated with cognitive functioning, but ideally also prove to be of added value when compared with these more readily available MRI markers. To reduce the number of variables in the linear regression analyses, a Bayesian approach was first used to determine which of the 18 white matter tracts were associated with cognitive functioning, as this method deals well with multicollinearity and multiple comparisons. Even so, a relatively high number of variables were included in the final regression models, which increases the risk of overfitting. We therefore additionally used a backward regression model in which only a few variables remained (indicating that the other variables were redundant), and these few variables always included MD of the strategic white matter tracts (Table 4). These complementary Bayesian and linear regression-based analyses provide converging evidence for an association between MD of the identified strategic white matter tracts and cognitive functioning. Still, the added value of a DTI-based assessment of these tracts on top of conventional MRI and global DTI markers needs to be reproduced in another memory clinic cohort before an attempt to translate these findings to clinical practice can be made. 
In summary, our findings suggest that the microstructural characteristics of strategic white matter tracts are functionally relevant MRI markers in memory clinic patients with vascular brain injury. The strategic white matter tract microstructure is of additional value in explaining variance in cognitive functioning when combined with both conventional MRI markers and the global white matter microstructure. Whether these associations are driven by (either vascular or neurodegenerative) brain injury or reflect healthy structure-function relations remains to be determined. Longitudinal studies are needed to determine whether a DTI-based assessment of strategic white matter tracts has any value in predicting the rate of cognitive decline and response to (pharmacological) treatment strategies.

\section{Acknowledgments}

Members of the Utrecht Vascular Cognitive Impairment (VCI) Study group involved in the present study (in alphabetical order by department): UMCU, The Netherlands, Department of Neurology: E. van den Berg, J.M. Biesbroek, G.J. Biessels, M. Brundel, W.H. Bouvy, L.G. Exalto, C.J.M. Frijns, O. Groeneveld, S.M. Heringa, N. Kalsbeek, L.J. Kappelle, Y.D. Reijmer, and J. Verwer; Department of Radiology/Image Sciences Institute: J. de Bresser, H.J. Kuijf, A. Leemans, P.R. Luijten, M.A. Viergever, K.L. Vincken, and J.J.M. Zwanenburg; Department of Geriatrics: H.L. Koek and J.E. de Wit; Hospital Diakonessenhuis Zeist, The Netherlands: M. Hamaker, R. Faaij, M. Pleizier, and E. Vriens.

\section{Disclosure Statement}

The authors report no conflict of interests.

\section{Funding Sources}

The work of A. Leemans is supported by Vidi Grant 639.072.411 from the Netherlands Organisation for Scientific Research (NWO). The work of G.J. Biessels is supported by grant 2010T073 from the Dutch Heart Association and Vidi grant 91711384 from ZonMw, The Netherlands Organisation for Health Research and Development.

\section{References}

1 Gorelick PB, Scuteri A, Black SE, Decarli C, Greenberg SM, Iadecola C, et al; American Heart Association Stroke Council, Council on Epidemiology and Prevention, Council on Cardiovascular Nursing, Council on Cardiovascular Radiology and Intervention, Council on Cardiovascular Surgery and Anesthesia: Vascular contributions to cognitive impairment and dementia: a statement for healthcare professionals from the American Heart Association/American Stroke Association. Stroke 2011;42:2672-2713.

2 Prins ND, Scheltens P: White matter hyperintensities, cognitive impairment and dementia: an update. Nat Rev Neurol 2015;11:157-165.

3 Baykara E, Gesierich B, Adam R, Tuladhar AM, Biesbroek JM, Koek HL, Ropele S, Jouvent E; Alzheimer's Disease Neuroimaging Initiative, Chabriat H, Ertl-Wagner B, Ewers M, Schmidt R, de Leeuw FE, Biessels GJ, Dichgans M, Duering M: A novel imaging marker for small vessel disease based on skeletonization of white matter tracts and diffusion histograms. Ann Neurol 2016;80:581-592.

4 Madden DJ, Bennett IJ, Burzynska A, Potter GG, Chen NK, Song AW: Diffusion tensor imaging of cerebral white matter integrity in cognitive aging. Biochim Biophys Acta 2012;1822:386-400.

5 Nitkunan A, Barrick TR, Charlton RA, Clark CA, Markus HS: Multimodal MRI in cerebral small vessel disease: its relationship with cognition and sensitivity to change over time. Stroke 2008;39:1999-2005.

6 van Norden AG, van Uden IW, de Laat KF, van Dijk EJ, de Leeuw FE: Cognitive function in small vessel disease: the additional value of diffusion tensor imaging to conventional magnetic resonance imaging: the RUN DMC study. J Alzheimers Dis 2012;32:667-676.

7 Viswanathan A, Godin O, Jouvent E, O’Sullivan M, Gschwendtner A, Peters N, Duering M, Guichard JP, Holtmannspötter M, Dufouil C, Pachai C, Bousser MG, Dichgans M, Chabriat H: Impact of MRI markers in subcortical vascular dementia: a multi-modal analysis in CADASIL. Neurobiol Aging 2010;31:1629-1636. 
8 Schiavone F, Charlton RA, Barrick TR, Morris RG, Markus HS: Imaging age-related cognitive decline: a comparison of diffusion tensor and magnetization transfer MRI. J Magn Reson Imaging 2009;29:23-30.

9 Biesbroek JM, Weaver NA, Biessels GJ: Lesion location and cognitive impact of cerebral small vessel disease. Clin Sci (Lond) 2017;131:715-728.

10 Duering M, Zieren N, Hervé D, Jouvent E, Reyes S, Peters N, Pachai C, Opherk C, Chabriat H, Dichgans M: Strategic role of frontal white matter tracts in vascular cognitive impairment: a voxel-based lesion-symptom mapping study in CADASIL. Brain 2011;134:2366-2375.

11 Boomsma JMF, Exalto LG, Barkhof F, van den Berg E, de Bresser J, Heinen R, Koek HL, Prins ND, Scheltens P, Weinstein HC, van der Flier WM, Biessels GJ: Vascular cognitive impairment in a memory clinic population: rationale and design of the "Utrecht-Amsterdam Clinical Features and Prognosis in Vascular Cognitive Impairment" (TRACE-VCI) Study. JMIR Res Protoc 2017;6:e60.

12 Fazekas F, Chawluk JB, Alavi A, Hurtig HI, Zimmerman RA: MR signal abnormalities at 1.5 T in Alzheimer's dementia and normal aging. AJR Am J Roentgenol 1987;149:351-356.

13 Deelman BG, Liebrand WB, Koning-Haanstra M, van den Burg W: Measurements of aphasic disorders. A brief description of the SAN-battery (in Dutch). Gerontologie 1980;11:17-21.

14 Corrigan JD, Hinkeldey NS: Relationships between parts A and B of the Trail Making Test. J Clin Psychol 1987; 43:402-409.

15 Miller DC: Best Practices in School Neuropsychology: Guidelines for Effective Practice, Assessment, and Evidence-Based Intervention. Hoboken, John Wiley \& Sons, 2009.

16 Houx PJ, Shepherd J, Blauw GJ, Murphy MB, Ford I, Bollen EL, Buckley B, Stott DJ, Jukema W, Hyland M, Gaw A, Norrie J, Kamper AM, Perry IJ, MacFarlane PW, Meinders AE, Sweeney BJ, Packard CJ, Twomey C, Cobbe SM, Westendorp RG: Testing cognitive function in elderly populations: the PROSPER study. PROspective Study of Pravastatin in the Elderly at Risk. J Neurol Neurosurg Psychiatry 2002;73:385-389.

17 Moses JA Jr, Pritchard DA, Adams RL: Neuropsychological information in the Wechsler Adult Intelligence Scale-Revised. Arch Clin Neuropsychol 1997;12:97-109.

18 Brand N, Jolles J: Learning and retrieval rate of words presented auditorily and visually. J Gen Psychol 1985; 112:201-210.

19 Verhage F: Intelligentie en leeftijd: Onderzoek bij Nederlanders van twaalf tot zevenenzeventig jaar. Assen, Van Gorcum, 1964.

20 Reijmer YD, Freeze WM, Leemans A, Biessels GJ; Utrecht Vascular Cognitive Impairment Study Group: The effect of lacunar infarcts on white matter tract integrity. Stroke 2013;44:2019-2021.

21 Wardlaw JM, Smith EE, Biessels GJ, Cordonnier C, Fazekas F, Frayne R, et al; Standards for Reporting Vascular Changes on Neuroimaging (STRIVE v1): Neuroimaging standards for research into small vessel disease and its contribution to ageing and neurodegeneration. Lancet Neurol 2013;12:822-838.

22 Ritter F, Boskamp T, Homeyer A, Laue H, Schwier M, Link F, Peitgen HO: Medical image analysis. IEEE Pulse 2011;2:60-70.

23 Ashburner J, Friston KJ: Unified segmentation. Neuroimage 2005;26:839-851.

24 Leemans A, Jeurissen B, Sijbers J, Jones D: ExploreDTI: a graphical toolbox for processing, analyzing, and visualizing diffusion MR data. Proc Intl Soc Mag Reson Med 2009;17:3537.

25 Leemans A, Jones DK: The B-matrix must be rotated when correcting for subject motion in DTI data. Magn Reson Med 2009;61:1336-1349.

26 Irfanoglu MO, Walker L, Sarlls J, Marenco S, Pierpaoli C: Effects of image distortions originating from susceptibility variations and concomitant fields on diffusion MRI tractography results. Neuroimage 2012;61:275288.

27 Jeurissen B, Leemans A, Jones DK, Tournier JD, Sijbers J: Probabilistic fiber tracking using the residual bootstrap with constrained spherical deconvolution. Hum Brain Mapp 2011;32:461-479.

28 Reijmer YD, Fotiadis P, Martinez-Ramirez S, Salat DH, Schultz A, Shoamanesh A, Ayres AM, Vashkevich A, Rosas D, Schwab K, Leemans A, Biessels GJ, Rosand J, Johnson KA, Viswanathan A, Gurol ME, Greenberg SM: Structural network alterations and neurological dysfunction in cerebral amyloid angiopathy. Brain 2015;138:179188.

29 Lebel C, Walker L, Leemans A, Phillips L, Beaulieu C: Microstructural maturation of the human brain from childhood to adulthood. Neuroimage 2008;40:1044-1055.

30 Scutari M: Learning Bayesian networks with the bnlearn R package. J Stat Software 2010;35:1-22.

31 Duering M, Gesierich B, Seiler S, Pirpamer L, Gonik M, Hofer E, Jouvent E, Duchesnay E, Chabriat H, Ropele S, Schmidt R, Dichgans M: Strategic white matter tracts for processing speed deficits in age-related small vessel disease. Neurology 2014;82:1946-1950.

32 Fujie S, Namiki C, Nishi H, Yamada M, Miyata J, Sakata D, Sawamoto N, Fukuyama H, Hayashi T, Murai T: The role of the uncinate fasciculus in memory and emotional recognition in amnestic mild cognitive impairment. Dement Geriatr Cogn Disord 2008;26:432-439.

33 Urger SE, De Bellis MD, Hooper SR, Woolley DP, Chen SD, Provenzale J: The superior longitudinal fasciculus in typically developing children and adolescents: diffusion tensor imaging and neuropsychological correlates. J Child Neurol 2015;30:9-20.

34 Jeurissen B, Leemans A, Tournier JD, Jones DK, Sijbers J: Investigating the prevalence of complex fiber configurations in white matter tissue with diffusion magnetic resonance imaging. Hum Brain Mapp 2013;34:27472766. 
Biesbroek et al.: Microstructure of Strategic White Matter Tracts and Cognition in Memory Clinic Patients with Vascular Brain Injury

35 Tax CM, Jeurissen B, Vos SB, Viergever MA, Leemans A: Recursive calibration of the fiber response function for spherical deconvolution of diffusion MRI data. Neuroimage 2014;86:67-80.

36 Nordlund A, Göthlin M, Wallin A: Vascular disease, Alzheimer's disease biomarkers and cognition in mild cognitive impairment: additive or synergetic effects? Dement Geriatr Cogn Disord 2011;32:250-256.

37 Turken A, Whitfield-Gabrieli S, Bammer R, Baldo JV, Dronkers NF, Gabrieli JD: Cognitive processing speed and the structure of white matter pathways: convergent evidence from normal variation and lesion studies. Neuroimage 2008;42:1032-1044.

38 Lawrence AJ, Patel B, Morris RG, MacKinnon AD, Rich PM, Barrick TR, Markus HS: Mechanisms of cognitive impairment in cerebral small vessel disease: multimodal MRI results from the St George's Cognition and Neuroimaging in Stroke (SCANS) study. PLoS One 2013;8:e61014.

39 Duering M, Gonik M, Malik R, Zieren N, Reyes S, Jouvent E, Hervé D, Gschwendtner A, Opherk C, Chabriat H, Dichgans M: Identification of a strategic brain network underlying processing speed deficits in vascular cognitive impairment. Neuroimage 2013;66:177-183.

40 Biesbroek JM, Kuijf HJ, van der Graaf Y, Vincken KL, Postma A, Mali WP, Biessels GJ, Geerlings MI; SMART Study Group: Association between subcortical vascular lesion location and cognition: a voxel-based and tract-based lesion-symptom mapping study. The SMART-MR study. PLoS One 2013;8:e60541.

41 Reijmer YD, Leemans A, Caeyenberghs K, Heringa SM, Koek HL, Biessels GJ; Utrecht Vascular Cognitive Impairment Study Group: Disruption of cerebral networks and cognitive impairment in Alzheimer disease. Neurology 2013;80:1370-1377.

42 Klunk WE, Engler H, Nordberg A, Wang Y, Blomqvist G, Holt DP, Bergström M, Savitcheva I, Huang GF, Estrada S, Ausén B, Debnath ML, Barletta J, Price JC, Sandell J, Lopresti BJ, Wall A, Koivisto P, Antoni G, Mathis CA, Långström B: Imaging brain amyloid in Alzheimer's disease with Pittsburgh Compound-B. Ann Neurol 2004;55: 306-319.

43 van Veluw SJ, Hilal S, Kuijf HJ, Ikram MK, Xin X, Yeow TB, Venketasubramanian N, Biessels GJ, Chen C: Cortical microinfarcts on 3T MRI: clinical correlates in memory-clinic patients. Alzheimers Dement 2015;11:15001509.

44 Clerici F, Caracciolo B, Cova I, Fusari Imperatori S, Maggiore L, Galimberti D, Scarpini E, Mariani C, Fratiglioni L: Does vascular burden contribute to the progression of mild cognitive impairment to dementia? Dement Geriatr Cogn Disord 2012;34:235-243.

45 Gattringer T, Enzinger C, Ropele S, Gorani F, Petrovic KE, Schmidt R, Fazekas F: Vascular risk factors, white matter hyperintensities and hippocampal volume in normal elderly individuals. Dement Geriatr Cogn Disord 2012;33:29-34

46 Duering M, Righart R, Wollenweber FA, Zietemann V, Gesierich B, Dichgans M: Acute infarcts cause focal thinning in remote cortex via degeneration of connecting fiber tracts. Neurology 2015;84:1685-1692.

47 Salthouse TA, Nesselroade JR, Berish DE: Short-term variability in cognitive performance and the calibration of longitudinal change. J Gerontol B Psychol Sci Soc Sci 2006;61:P144-P151. 